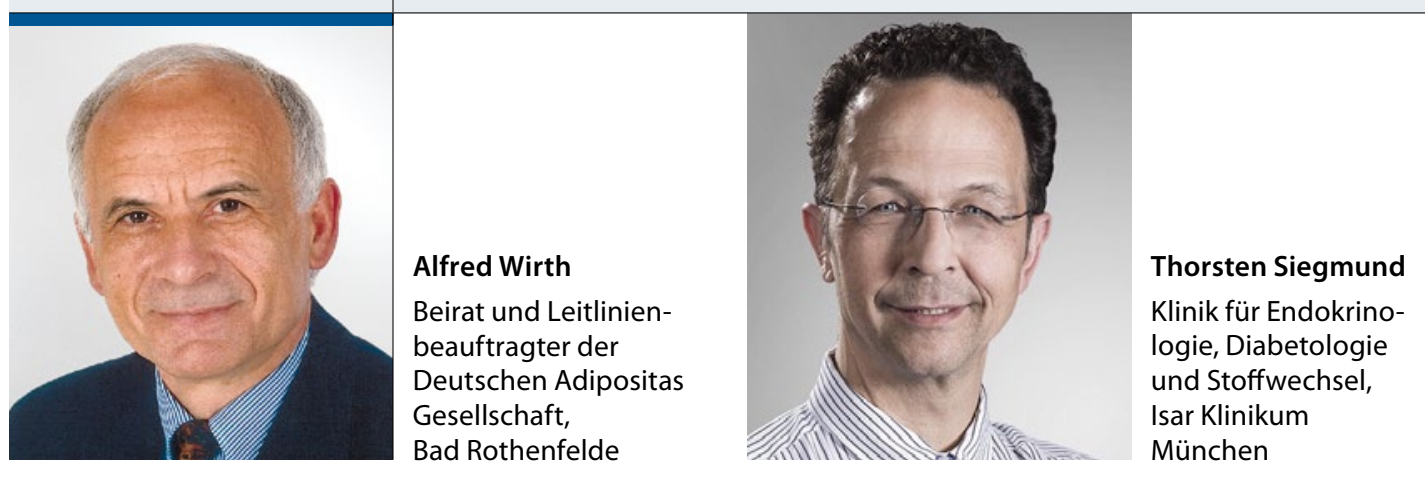

\title{
Thrombosen bei Adipositas und Wirkung neuer Antidiabetika
}

\section{Bei Adipositas an Thrombosen und Thromboembolien denken!}

Die Wahrnehmung der Adipositas als Krankheit hat in den letzten Jahren sowohl bei Betroffenen als auch beim medizinischen Fachpersonal zugenommen. Von den etwa 20 Krankheiten, die mit der Adipositas evidenzbasiert assoziiert sind, steht die Thromboembolie ziemlich weit hinten, obwohl diese Komplikation bei Adipositas um das Doppelte erhöht ist. Zum einen begünstigen Parameter der Gerinnung und Fibrinolyse die Entstehung von Thromben, zum anderen sind es mechanische Faktoren wie der erhöhte intraabdominale Druck, der auch die Entwicklung eines gastroösophagealen Refluxes und die Entstehung einer Hypertonie begünstigt.

Adipöse leiden oft auch an Krankheiten wie einer Herzinsuffizienz oder an Immobilität, was ebenfalls thrombosesteigernd wirkt. Selbst wenn schon Beschwerden und Symptome (z. B. geschwollenes Bein) für eine Thrombose oder Thromboembolie vorliegen, ist die Wahrnehmung des Betroffenen eingeschränkt und die Diagnostik (z. B. Sonografie) erschwert.

Die Therapie mit Antikoagulanzien wirft viele Fragen auf, da die Pharmakokinetik bei Adipositas in mehrfacher Hinsicht verändert sein kann: die Resorption (z.B. nach bariatrischer Operation), die Verteilung im Körper (erhöhtes Plasmavolumen, vermehrtes Körperfett) und die Ausscheidung (z. B. nach maldigestiver Operation). Da Antikoagulanzien mit unterschiedlicher Pharmakokinetik zur Anwendung kommen, ist eine optimale Dosierung nicht einfach.

Es wundert daher nicht, dass viele Patienten unterdosiert sind; gelegentlich kommen auch Überdosierungen vor. Der Beitrag hilft dem klinisch Tätigen, eine individuelle Dosis für das jeweilige Antikoagulans zu finden.

\section{Mikrovaskuläre Schäden durch Antidiabetika verbessern}

Eine Optimierung des Glukosestoffwechsels ist eng assoziiert mit der Reduktion mikrovaskulärer Folgeschäden, unter anderem an der Niere.

Dass die Prognose von Menschen mit Diabetes durch eine rein glukosezentrische Vorgehensweise nicht ausreichend zu verbessern ist, ist bekannt. Nur wenn alle relevanten Risikofaktoren gleichzeitig optimiert werden, werden harte Endpunkte relevant reduziert. In den letzten 15 Jahren versuchten die Mediziner mit den vorhandenen Möglichkeiten (Lifestyle und medikamentöse Therapieoptionen) diese Prognose zu verbessern. Die erzielten Fortschritte wurden eher „evolutionär“, d.h. durch kleinere Modifikationen in der Herangehensweise, im konsequenteren Einsatz beziehungsweise in der Kombination von Medikamenten erzielt.

Erst durch die Daten aus Sicherheitsstudien der zuletzt eingeführten Antidiabetika, der SGLT2-Inhibitoren und GLP1-Rezeptoragonisten, bahnt sich nun eine Art Revolution an. Es ist noch nicht vollständig verstanden, wie die Substanzen Empagliflozin und Liraglutid kardiovaskuläre Endpunkte und vor allem die Mortalität reduzieren. Jetzt sind darüber hinaus neue Daten verfügbar, die einen zusätzlichen Benefit auch für mikrovaskuläre Folgeschäden an der Niere zeigen. Diese Effekte kommen additiv zu den Vorteilen einer RAAS-Blockade hinzu.

Diese Vorteile sind nicht durch die reine Glukosesenkung erklärbar, was die Substanzen auch für Patienten ohne Diabetes mellitus interessant machen dürfte. So wurden z. B. erste Studien bei Menschen mit chronischer Niereninsuffizienz ohne Diabetes angestoßen. Zu prüfen ist auch, ob der kardiovaskuläre Hochrisikopatient ohne Diabetes durch diese Substanzen profitieren könnte.

Alfred Wirth und Thorsten Siegmund 